\title{
Nonimmune fetal hydrops and lysosomal storage disease: the finding of vacuolated lymphocytes in ascitic fluid in two cases
}

\author{
R. B. Dugan ${ }^{1 *}$, M. A. Pletneva ${ }^{2}$, K. Salari $^{1}$, D. R. Berman ${ }^{1}$ and M. C. Treadwell ${ }^{1}$ \\ ${ }^{1}$ Fetal Diagnostic Center, University of Michigan Health System, Ann Arbor, MI, USA \\ ${ }^{2}$ Department of Pathology, University of Michigan Health System, Ann Arbor, MI, USA \\ *Correspondence to: R. B. Dugan. E-mail: rbdugan@comcast.net
}

Funding sources: None

Conflicts of interest: None declared

Nonimmune fetal hydrops (NIFH) describes the abnormal accumulation of fluid in at least two fetal compartments, including soft tissue edema, or fluid accumulation in body cavities such as ascites, pleural effusion, or pericardial effusion. Polyhydramnios and placentomegaly frequently accompany fetal hydrops. NIFH is a heterogeneous condition and establishing a diagnosis can be challenging. Bellini et al. ${ }^{1}$ reviewed and classified causes of NIFH in order of prevalence: cardiovascular disorders (21.7\%), chromosome imbalances (13.4\%), hematologic abnormalities (10.4\%), infections (6.7\%), intrathoracic masses (6.0\%), lymph vessel dysplasias (5.7\%), twin-to-twin transfusions and placental causes (5.6\%), syndromic (4.4\%), urinary tract malformations $(2.3 \%)$, inborn errors of metabolism (1.1\%), extra-thoracic tumors $(0.7 \%)$, gastrointestinal disorders $(0.5 \%)$, miscellaneous causes $(3.7 \%)$, and idiopathic (17.8\%). After excluding immune causes of fetal hydrops, the search for an underlying cause of NIFH may be extensive and include ultrasound, fetal echocardiogram, evaluation for infectious etiologies, and invasive testing for fetal karyotype. What constitutes appropriate work-up beyond this initial investigation is controversial, but could include screening for metabolic conditions, such as lysosomal storage disease (LSD) via enzyme analysis in cultured cells from amniocytes or fibroblasts, microarray analysis to detect microdeletions or duplications, or single gene testing (e.g. Noonan syndrome). Sequential protocols for investigation of NIFH have been proposed to optimize diagnosis and avoid cost prohibitive investigations. ${ }^{1-3}$ Despite these efforts, such protocols are not universally practiced. It remains essential to establish diagnostic algorithms for NIFH, which optimize resources and to define appropriate indications for metabolic disease testing. Key to these efforts is identifying factors that may facilitate a specific prenatal diagnosis in these complicated cases resulting in improved detection rates and allowing providers to better care for and counsel their patients. We present two cases of NIFH caused by underlying lysosomal storage disease: galactosialidosis (case 1) and mucopolysaccharidosis type VII (case 2). The in utero presentation and the unique finding of vacuolated lymphocytes in fetal peritoneal fluid lead to the diagnosis.

Patient 1, a 27 year old G1P0 female, presented to our institution at $252 / 7$ weeks gestation due to known NIFH. Family history was noncontributory/without consanguinity. Fetal ascites was first suspected at 18 weeks gestation during routine fetal survey. Follow-up ultrasound at 19 5/7 weeks revealed fetal ascites and ventricular septal defect. Concurrent amniocentesis revealed normal male karyotype (46,XY), normal amniotic fluid alpha-fetoprotein at $1.3 \mathrm{MoM}$, normal single nucleotide polymorphism microarray analysis, and negative PCR for parvovirus B19, cytomegalovirus, varicella zoster virus, herpes simplex virus, and Toxoplasma gondii. Ultrasound at 25 weeks gestation revealed a hydropic fetus with appropriate biometry, massive ascites, scalp edema, mild ventriculomegaly, cardiomegaly with a ventricular septal defect, dilated loops of bowel with dilated rectum, placentomegaly, and normal Doppler velocimetry in umbilical and middle cerebral arteries. At 30 1/7 weeks of gestation, peak systolic velocities of the middle cerebral artery were elevated at 1.55 MoM. Given this and further progression of fetal hydrops of unknown etiology, the patient underwent ultrasound guided cordocentesis with fetal blood transfusion for mild anemia (hematocrit 30\%). A fetal paracentesis was performed to remove $500 \mathrm{cc}$ of ascites because of concern that the upward compression of the diaphragm by the ascites contributed to the worsening hydrops. Review of fetal ascitic fluid revealed predominantly histiocytes (93\%) and rare vacuolated lymphocytes. Despite initial improvement in hydrops, reaccumulation occurred. Repeat fetal paracentesis removing $300 \mathrm{cc}$ was undertaken to decompress the abdomen and facilitate vaginal delivery prior to induction at 36 weeks of gestation (head circumference to abdominal circumference ratio was 0.73). A $2240 \mathrm{~g}$ male infant was delivered with Apgar scores of 2 and 5 at 1 and $5 \mathrm{~min}$. Physical exam revealed poor 
respiratory effort requiring intubation, abdominal wall laxity, skin edema, and low-set ears. Marked cytoplasmic vacuolization was seen in lymphocytes in ascites fluid (Figure 1) sent immediately prior to delivery and within placenta, suggestive of a metabolic storage disease. Enzyme screening panel for LSD revealed low beta-galactosidase activity in leukocytes $(8.5 \mathrm{nmol} / \mathrm{hr} / \mathrm{mg}$ protein, approximately $10 \%$ of normal) with normal activity in plasma, and high sialic acid content in leukocytes with normal free sialic acid content, resulting in diagnosis of galactosialidosis. The infant was extubated on day of life 17, discharged to home at 1 month of age, and is currently receiving home hospice services.

Patient 2 is a 33 year old G1P0 female whose routine fetal anatomy scan at $191 / 7$ weeks of gestation revealed skin edema, bilateral pleural effusions, and ascites. Family history was noncontributory/without consanguinity. Amniocentesis revealed normal male karyotype $(46, \mathrm{XY})$, normal oligonucleotide array, and negative work-up for cytomegalovirus, parvovirus, and toxoplasmosis. Patient was referred at 21 6/7 weeks of gestation for second opinion and ultrasound confirmed the prior findings. Fetal thoracentesis was performed to evaluate pleural effusions for lymphocytes and to consider if thoracic shunting would be a potential therapy for this fetus. The pleural fluid was predominantly reactive histiocytes; patient continued the pregnancy without further fetal interventions. Ultrasound at 22 $6 / 7$ weeks revealed reaccumulation and worsening of pleural effusions, skin edema, and hydroceles, with normal umbilical and middle cerebral artery Doppler velocimetries. Subsequent ultrasounds revealed stable hydrops. At 36 weeks of gestation, abnormal antenatal testing lead to cesarean delivery of a $3740 \mathrm{~g}$ male infant with Apgars of 6 and 7 at 1 and 5 min. Paracentesis (620 mL removed) was performed prior to delivery to facilitate resuscitation and ease of delivery. Review of fetal ascites fluid

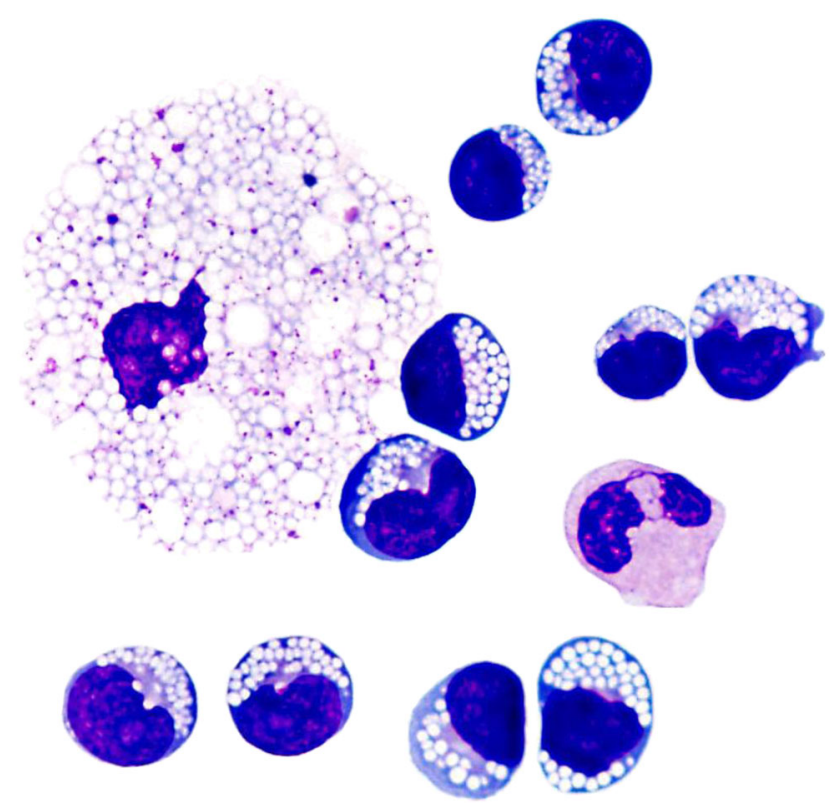

Figure 1 Marked vacuolization of histiocytes and lymphocytes in ascites derived from fetus with galactosialidosis. Photograph taken at 1000x magnification (oil immersion) revealed metachromatic cytoplasmic granules within leukocytes of all types (Alder-Reilly anomaly, Figure 2), suggestive of mucopolysaccharidosis (MPS). Placental histopathology showed marked fetal obliterative vasculopathy with extensive villous avascularity, vascular myonecrosis, and villous edema. Urine studies revealed abnormal excretion of mucopolysaccharides. Enzymatic analysis showed significantly reduced beta glucuronidase activity in leukocytes consistent with a diagnosis of MPS VII (Sly syndrome). GUSB gene sequencing revealed heterozygosity for two previously unreported variants (c.148G $>$ T and c.1824C $>$ G). Both changes are predicted to result in pathogenic amino acid substitutions. The infant was discharged home on day of life 40 with nasogastric tube feeds and is followed by palliative care team.

Nonimmune fetal hydrops is a heterogeneous condition. In the majority of cases, inborn errors of metabolism will not be considered until there are multiple affected pregnancies. Several LSDs have been diagnosed following the presentation of NIFH. We present two cases of NIFH due to LSD diagnosed in a first pregnancy highlighting the unique circumstances that led to a diagnosis. In both cases, the ultrasound findings of NIFH included marked ascites. Massive ascites has previously been reported as a key finding in in utero manifestation of sialic acid and other LSDs. ${ }^{4}$ In contrast, cases of Noonan syndrome are more likely to present with increased nuchal translucency, cystic hygroma, and hydrothorax. ${ }^{5}$ A greater

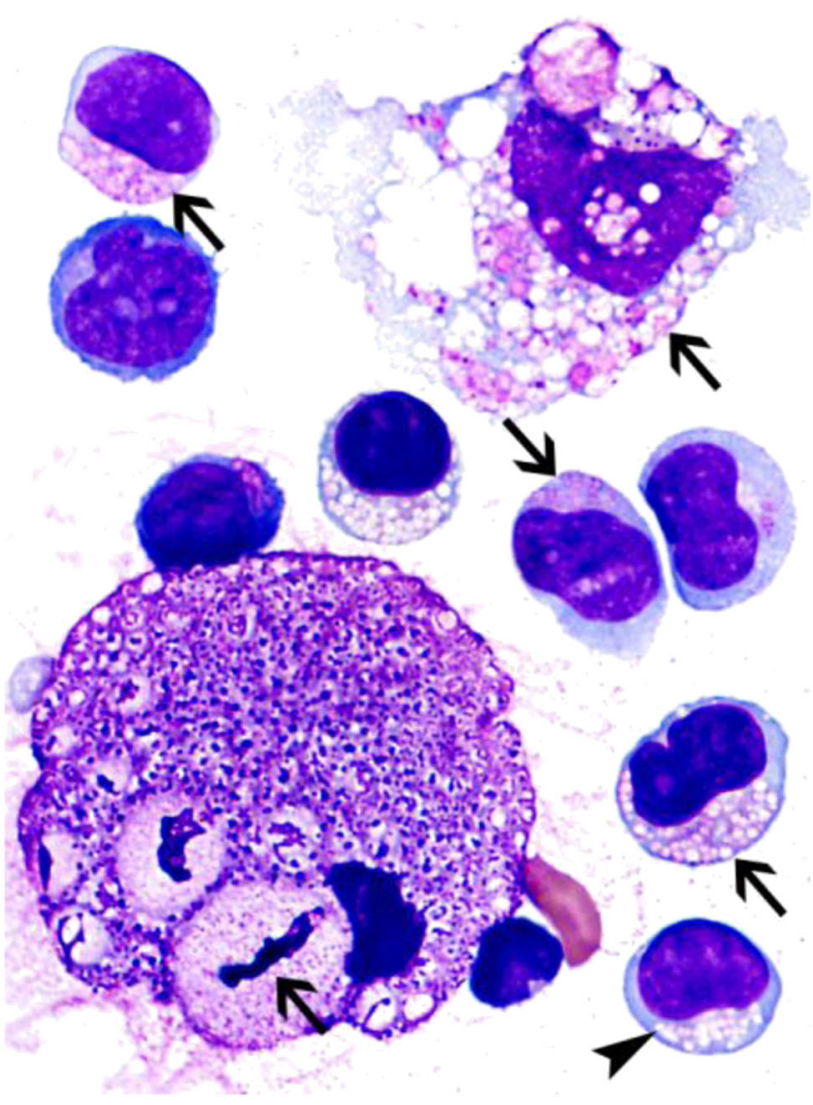

Figure 2 Alder-Reilly (chunky deeply asurophilic) bodies and metachromatic inclusions (arrows) and vacuoles (arrowhead) in histiocytes and lymphocytes in ascites derived from fetus with MPS VII. Photograph taken at $1000 \times$ magnification (oil immersion) 
awareness of the presentation of the more rare etiologies for NIFH may help guide prenatal testing when the more common causes have been eliminated. This may be particularly useful when prenatal samples are limited. Furthermore, the diagnosis, once made in a family, allows for mutation analysis and prenatal DNA testing in future pregnancies.

In both of the presented cases, the initial investigation into the etiology of NIFH was negative, and testing for inborn errors of metabolism was not included. Fluid samples obtained from therapeutic procedures in both cases contributed to the early diagnosis following delivery. Vacuolization in fetal cells of placentas $^{6}$ and circulating leukocytes ${ }^{7}$ has been well documented in cases of infantile LSD. Vacuolated lymphocytes were reported in ascites of a newborn delivered at 31 4/7 weeks of gestation. ${ }^{8}$ To our knowledge, ours are the first reported cases of vacuolization in lymphocytes obtained via fetal paracentesis. Cytologic evaluation of paracentesis fluid at 30 $1 / 7$ weeks of gestation (case 1) revealed a low lymphocyte count (4\%) with few vacuolated lymphocytes noted. However, ascites obtained at 36 weeks of gestation contained significantly more lymphocytes (63\%) with marked cytoplasmic vacuolization suggestive of an underlying LSD. All differentiated blood cell types, including lymphocytes, are represented in peripheral circulation by 30 weeks of gestation; however, their proportions continue to change throughout the remainder of pregnancy and the neonatal period. ${ }^{9}$ Whether the difference in content of vacuolated lymphocytes in successive paracentesis fluids reflects sampling error or in utero progression of disease remains unknown, but gestational age could alter the diagnostic utility of testing the ascitic fluid. In case 2, cytologic evaluation of fetal ascites obtained at 36 3/ 7 weeks of gestation revealed Alder-Reilly bodies within histiocytes and metachromatic granules and vacuoles within lymphocytes, suggestive of mucopolysaccharidosis. Placental examination showed marked damage of fetal vasculature. We are unaware of reports of vacuolization at earlier gestational ages. In a case report of NIFH associated with MPS VII that resulted in intrauterine fetal demise at 16 weeks, no abnormal inclusions or vacuolization of blood cells or placenta were noted. ${ }^{10}$

In conclusion, we report two cases of NIFH due to lysosomal storage disease: galactosialidosis and mucopolysaccharidosis type VII. In both cases, massive ascites was a significant finding at presentation. Cytologic evaluation of fetal ascites revealed vacuolated lymphocytes, guiding further testing and expediting postnatal diagnosis. Histopathologic examination of fetal ascites may be useful in cases in which paracentesis is performed for therapeutic reasons, and etiology for the ascites is unknown. In our cases, abnormal vacuolization was seen in the third trimester. Second trimester pathology in fetal ascites may not be as reliable, possibly due to evolution of the storage disease or natural history of the ascites.

\section{WHAT'S ALREADY KNOWN ABOUT THIS TOPIC?}

- Nonimmune fetal hydrops (NIFH) is a heterogeneous condition and establishing a diagnosis can be challenging

- Inborn errors of metabolism are one of many possible etiologies for NIFH.

- Inborn errors of metabolism are often missed in utero.

\section{WHAT DOES THIS STUDY ADD?}

- We present two cases of NIFH caused by underlying lysosomal storage disease: galactosialidosis and mucopolysaccharidosis type VII.

- The unique finding of vacuolated lymphocytes in fetal peritoneal fluid lead to the diagnosis.

\section{REFERENCES}

1. Bellini C, Hennekam RCM, Bonioli E. A diagnostic flow chart for nonimmune hydrops fetalis. Am J Med Genet Part A 2009;149A:852-3.

2. Kooper AJA, Janssens PMW, de Groot ANJA, et al. Lysosomal storage diseases in non-immune hydrops fetalis pregnancies. Clin Chim Acta 2006;371:176-82.

3. Gort L, Granell MR, Fernandez G, et al. Fast protocol for the diagnosis of lysosomal diseases in nonimmune hydrops fetalis. Prenat Diagn 2012;32:1139-42.

4. Froissart R, Cheillan D, Bouvier R, et al. Clinical, morphological, and molecular aspects of sialic acid storage disease manifesting in utero. J Med Genet 2005;42:829-36.

5. Baldassarre G, Mussa A, Dotta A, et al. Prenatal features of Noonan syndrome: prevalence and prognostic value. Prenat Diagn 2011;31:949-54.
6. Benirschke K, Burton GJ, Baergen RN. Pathology of Human Placenta. Berlin: Springer, 2012.

7. Anderson G, Smith VV, Malone M, Sebire NJ. Blood film examination for vacuolated lymphocytes in the diagnosis of metabolic disorders; retrospective experience of more than 2500 cases from a single centre. J Clin Pathol 2005;58:1305-10.

8. Sergi C, Beedgen B, Kopitz MB, et al. Refractory congenital ascites as a manifestation of neonatal sialidosis: clinical, biochemical and morphological studies in a newborn Syrian male infant. Am J Perinatol 1999;16(3):133-41.

9. Hann IM, Gibson BES, Letsky EA. Fetal and Neonatal Haematology (1st edn), London: Bailliere Tindall, 1991.

10. Venkat-Raman N, Sebire MJ, Murphy KW. Recurrent fetal hydrops due to mucopolysaccharidosis type VII. Fetal Diagn Ther 2006;21:250-4. 